\title{
13. COMMISSION DES ECLIPSES DE SOLEIL
}

\section{Président: Prof. S. A. Mitchell, Leander McCormick Observatory, University of Virginia, Charlottesville, Va, U.S.A.}

Membres: MM. Abetti, Allen, Carroll, Clemence, Dunham, Fesenkov, Freundlich, Grönstrand, Horn d'Arturo, A. Hunter, J. Jackson, Lindblad, Link, Lyot, Menzel, Mikhailov, Millman, Minnaert, Smiley, J. Q. Stewart, Stratton, Van Biesbroeck, Woolley.

Since the date of the 1938 Stockholm meeting, there has been vast progress from eclipses both on the observational and the theoretical sides. In the interval there have been five total eclipses: 1940, October I; I94I, September 2I; I944, January 25; I945, July 9; and 1947, May 20. In spite of the war and of the ever-present enemy of eclipse observers, clouds, valuable observations were secured from each of the five eclipses.

It is becoming increasingly difficult to draw a line of delimitation between the work of Commission I3 (Eclipses) and of Commission II (Chromospheric Phenomena) and Commission I2 (Solar Radiation and the Solar Spectrum). Spectacular developments by the use of coronagraphs at the high-altitude stations in France, in Switzerland and in the United States (Harvard Station at II,532 ft.) and also by the improved motion-picture techniques developed at the McMath-Hulbert and the Mt Wilson Observatories have made it possible for refined observations of prominences and of the corona to be made outside of a total eclipse. As a splendid example of observations made within and outside the band of totality of an eclipse, may be mentioned the important publication by Waldmeier (Astron. Mitt. Zürich, No. I49, 1947) concerning coronagraph observations at Arosa on July 8-Io, I945, or, in other words, on the day of the total eclipse of July 9, and the preceding and following days.

Chromosphere. The most extended observations of the flash spectrum published in the past ten years are those of Mitchell ( $A p . J$. I05, I, I947), and the discussion of these observations by Wildt (loc. cit. p. 36). A total of 3500 spectral lines are recorded between the wave-lengths 3066 and $8863 \mathrm{~A}$. The intensities are given for the Sun and for the chromosphere, the heights above the photosphere in $\mathrm{km}$., and the excitation potentials. The observations are the result of ten eclipse expeditions. Another important contribution is that of Redman (M.N. 102, I34 and I42, 1942) which discussed the observations from the I940 eclipse. Eclipse observers with their temporary installations are usually at the mercy of great temperature changes which may cause large fluctuations in focus. Redman adopted the excellent plan of protecting the sensitive parts of his spectrograph by placing them vertically in a pit.

The Hydrogen Chromosphere. The prominence of the Balmer and the Paschen series of hydrogen is a manifestation of the overwhelming abundance of hydrogen in the solar atmosphere. The determination of the density gradient of hydrogen is a most important problem for the reason that it should reveal the structure of the bulk of the chromosphere. Heretofore, the chief investigators have been Pannekoek and Minnaert (Verh. Kon. Akad. Amsterdam, Vol. 13, No. 5, I928) and Cillié and Menzel (Harvard Obs. Circ. No. 410, 1935). Both discussions are based on the total intensities of the Balmer lines in the flash spectrum and these intensities are difficult to interpret on account of the self-absorption that may occur in the Balmer lines. The method adopted by Mitchell and Williams (AP. J. 77, I933) and by Wildt of utilizing the heights of the Balmer lines estimated from the tips of slitless curved lines turns out to be practically free from interference by the effect of self-absorption. The results attained agree closely with the analysis of the composition of the solar atmosphere of B. Strömgren (Festschrift für E. Strömgren, p. 214, I940).

In the solar chromosphere the last observed member is $n=37$ in the Balmer series and $n=40$ in the Paschen series. Wildt finds the results from both series in good accord. Taking the mean, he finds that in a chromosphere slab at height $500 \mathrm{~km}$. the number per cubic centimetre of positive ions is $2.4 \times \mathrm{IO}^{11}$.

Metals in the Chromosphere. Although some metallic lines in the chromosphere like 
the $\mathrm{H}$ and $\mathrm{K}$ lines of $\mathrm{Ca}$ II attain greater heights than hydrogen, while other enhanced lines of $\mathrm{Sr}, \mathrm{Ti}, \mathrm{Ba}$ and $\mathrm{Fe}$ attain comparable heights, the vast majority of metallic lines are below the $2000 \mathrm{~km}$. level. As was pointed out by Wildt (loc. cit.) and earlier by Mitchell and Williams ( $A p . J .77$, I, I933), the comparatively small range in the estimated heights has been a handicap in the determination of the density distribution of the metals in the chromosphere.

In consequence of the much greater range in the intensities of the metallic lines, Mitchell, when preparing for the I947 publication, made re-estimates of his spectra obtained at different eclipses and at the same time made estimates at heights of 400,600 , $800,1000,1200,1500,2000 \mathrm{~km}$. and on up to the highest levels reached. As explained in the publication, continued attempts were made by him to keep the scale of intensities as constant as possible. Entirely after the completion of the estimates, it was interesting to ascertain what the scale of estimates actually was. Consequently, the metallic lines were grouped into multiplets and then arranged according to doublets, triplets and so forth up to septets. (I trust the members of the Commission will pardon its president for enumerating here his findings.) For the sake of brevity, attention will be confined to the lines of doublets and triplets for the twofold reason that they involve the strongest lines and also that the theoretical intensities are more thoroughly determined. In addition to the intensities given in the 1947 publication, the estimates for individual lines at the $400,600 \mathrm{~km}$. and higher levels were all grouped together, but with diminished weights at the greater heights. Comparisons were made with Russell's important publication $(A p . J .83,129,1936)$. As the intensities, both observed and theoretical, are relative, the simplest method of procedure is to take the intensity of each line of the multiplet with respect to the total intensity in the multiplet as unity. Then for further simplicity, attention may be confined to the strongest line in each multiplet and its intensity relative to the multiplet as a whole as unity. With 26 doublets and 16 triplets to combine into averages, it was found that the ratio of the scales of the observed relative to the theoretical intensities was $100.4 \%$ for the doublets, and 100.8\% for the triplets, or $100.5 \%$ for the 42 multiplets. The theoretical scale derived from multiplet structure gives the logarithm of the number of 'active atoms' involved. Mitchell's observed scale for the stronger lines of the chromosphere agrees very closely with the logarithm of the square root of the number of active atoms. The agreement with the theoretical scale from multiplet structure is remarkably close. It might be added that scales of intensities are logarithmic and hence are similar to stellar magnitude scales.

In addition to the estimates of intensity made by Mitchell, his I930 photographs of the flash spectrum have been measured by Mulders with the micro-photometer at the Mount Wilson Observatory. It is therefore planned to derive curves of growth of spectral lines at different heights in the chromosphere. By this means, further information will be derived regarding the relative abundances of the metals and their density distribution.

Wave-Lengths in Emission and Absorption. Comparison by Redman between 328 emission lines with 159 absorption lines near the Sun's limb gives a mean shift in the sense absorption minus emission of $-0.0007 \pm 0.002 \mathrm{~A}$.

The Relative Abundance of the Metals. The discussion by Wildt of the relative number of atoms in the chromosphere (loc. cit. p. 74) is in good agreement with the earlier values in the chromosphere found by Menzel and by Mitchell and Williams and these values in turn with the results of Russell $(A p . J .70$, II, I929) for the Fraunhofer spectrum. The chromosphere is much richer in hydrogen than the photosphere. Although the metals differ comparatively little from each other in their abundances, Wildt finds that at the $500 \mathrm{~km}$. level, hydrogen is a million times more abundant than neutral iron.

Turbulent Pressure in the Chromosphere. McCrea's theory (M.N. 89, 7I8, I929) seemed to have been abundantly verified by various eclipse observers. According to Unsöld (Physik der Sternatmosphären, p. 437, I938) we read that the profiles of the chromosphere lines are mainly determined by turbulence, all of them being equally wide. He concludes that the line profiles measured by Pannekoek and by Minnaert, and also by Menzel (Publ. Lick Obs. 7, I, I93I) indicate turbulent velocities of the order of IO-I5 km./sec. 
Mitchell and Williams likewise find turbulent velocities confirmed by the relation between intensity and height. In a most surprising way, the results obtained by Redman, and independently by Wildt, have shown that we must abandon our previous model and that at least the lower parts of the chromosphere are practically in diffusion equilibrium, each gas showing the gradient corresponding to its molecular weight. The opinion expressed by Redman (M.N. 104, 103, I944) is: 'The present evidence then is that any motion in the solar reversing layer and lower chromosphere which can properly be called "turbulent", i.e. which is common to all material and not Maxwellian in distribution, does not exceed an average velocity of $\mathrm{I} \mathrm{km}$./sec. in any one co-ordinate.' From the solar curve of growth, K. O. Wright ( $A p . J .99,249$, I944) finds a turbulent velocity of $0.9 \mathrm{~km}$. $/ \mathrm{sec}$., while from Menzel and Rubenstein's curve the corresponding value is $0.6 \mathrm{~km}$./sec. Similarly, Struve and Elvey find that turbulence in reversing layers, estimated from curves of growth, must be less than $2 \mathrm{~km}$. $/ \mathrm{sec}$.

It should be added that it is difficult to reconcile these small turbulent motions with the classical picture of the sun as a 'prairie in flames', the presence of numerous small prominences in continual motion, with the very large hydrogen velocities from flares, and with the newly discovered spicules (Roberts, $A P . J$. Ior, 136, I944).

Temperatures in the Chromosphere. All methods of investigating the reversing layer have been in agreement in assigning temperatures of $4000^{\circ}$ to $6000^{\circ}$. The presence of He II, $4686 \mathrm{~A}$. in the flash spectrum has been difficult to explain. With temperatures of a million degrees demanded by the spectrum of the corona, it is evident that our ideas of the temperatures in the chromosphere and the corona must be radically revised.

For the chromosphere, there are three different methods of deriving temperatures: (I) excitation temperatures, (2) line profiles, and (3) height gradients. Among the more recent investigators of excitation temperatures are Wildt (loc. cit.), Goldberg ( $A p . J$. 90, 4I4, I939) and Petrie (J.R.A.S. Canada, 38, I37, I944). The temperatures for hydrogen are about $5000^{\circ}$, for helium the range is from $4300^{\circ}$ to $6700^{\circ}$ and for the metals the range is from $3000^{\circ}$ to $5000^{\circ}$. In other words, all authors are agreed in assigning approximately $5000^{\circ}$ as the excitation temperatures. In sharp contrast are the temperatures of $30,000^{\circ}$ found by Redman from line profiles. Starting with the height gradients determined by Wildt, Minnaert has calculated the (unpublished) temperatures of $35,000^{\circ}$ for $\mathrm{H} ; 60,000^{\circ}$ increasing with height to $100,000^{\circ}$ for $\mathrm{He}$; and $30,000^{\circ}$ increasing to $175,000^{\circ}$ for iron and the metals. The temperatures determined from gradients at $2000 \mathrm{~km}$. for hydrogen and the metals are in fair agreement with the kinetic temperatures from line profiles at the same elevations. In contrast, the excitation temperatures are much lower.

Eclipse Spectra from Airplanes. Flying under the auspices of R.C.A.F., Millman $(A . J$. $\mathbf{5 2}$, I57, I947) secured photographs at the I945 eclipse. All cameras were on fixed mounts, exposures being made electrically by remote control at intervals of $2-4$ sec. Pilots kept the aircraft on the correct course by using an adaptation of the reflector gunsight. Although some vibrations were evident, it is considered that this could have been eliminated had more time been available for mounting the instruments. Satisfactory low-dispersion coronal and chromospheric spectrograms were secured from elevations of 26,000 and 33,000 feet.

Future Chromospheric Observations. It is certain that the chromosphere cannot be in thermodynamic equilibrium. Hence the values of the 'temperature' calculated by applying equilibrium theory to such different properties as excitation, line profiles and density gradients may be expected to differ. The apparent variation in density at different heights in the chromosphere is such as would be produced if higher and higher temperatures were reached at greater and greater distances from the Sun's surface. The only method of solving the dilemma seems to be for future eclipse observers to take up the difficult task of deriving simultaneous observations of line profiles at different heights in the chromosphere.

Several of the more active members of the Commission, who have been interested in spectroscopic problems rather than in direct photography, have independently requested that a statement should be made in the report that after fifty years of photographic 
observations of eclipses there is little need of continuing routine spectrographic work on the flash spectrum on the short wave-length side of $7000 \mathrm{~A}$. At the red end, observations could be extended by the use of improved Eastman $Z$ plates, by lead sulphide photoelectric cells and by phosphors. Attention of possible observers should be called to the thinning out of chromospheric lines in this region and also to the presence of strong atmospheric absorption bands.

The Corona. A dozen years ago it used to be said that the solar corona could be observed only for an average of 60 seconds, or I minute of time per year, and exclusively at the time of a total eclipse. As a result primarily of the brilliant work of Lyot, it is nowadays possible to photograph the corona in broad daylight and continuously for several hours in succession on many days during the year when the air transparency is high.

The construction of the Lyot coronagraph with its filters and polarizers is described in several journals, including $A p . J$. ror, 255 , 1945. With the use of a radiation-separator, four images are produced corresponding to different radiations. Three of these are received on a moving film in size $24 \times 36 \mathrm{~mm}$. and they register simultaneously: (I) the prominences photographed in $\mathrm{H}_{\alpha}$ light, (2) the corona in the $6374 \mathrm{~A}$. line in the red, and (3) the corona in the green at $5303 \mathrm{~A}$. The fourth image is used for guiding purposes. Each of the three regions has a spectral width of $2 \mathrm{~A}$. Some of these films were continued for more than I2 hours and cover a total duration of 92 hours of action on the Sun. Spectra taken with concave grating $(A P . J \cdot 75, \mathrm{I}, \mathrm{I932})$ showed vast differences in structure between the 5303 and 6374 lines. The Lyot films 'confirm the difference between the green and the red corona and the nearly total independence of the corona and the prominences. On the other hand, they are far from confirming the motions in the corona mentioned by some observers: even when speeded up 2400 times, the corona remains stationary. The arches and jets appear and fade progressively along invisible trajectories, and the corona thus modifies its shape without perceptible motions.'

By the use of his fringe-photometer, Lyot was enabled to compare the intensities of the green and red lines with the intensity of polarized light. Sometimes the two lines have a sharp maximum near the same regions, but far more generally their maxima are at very different position angles. Near the Sun's poles, the intensity may be less than two-millionths of the continuous spectrum of the Sun, while near the equator the brightness may be 40 times greater.

Since 1943 , Lyot has been able to measure visually, in $5^{\circ}$ steps around the Sun's disk, the intensities of the radiations 5303,6374 and 5694 (when the activity is great), and these measures are compared with the quantity of polarized light, which in turn is sensibly proportional to the intensity of the continuous spectrum. These measures are continued as regularly as possible from the station at Pic du Midi, and they are utilized as advance predictions for observations on the ionosphere.

As a result of difference in the distribution of the spectral lines around the Sun and their connections with sunspots, Lyot (M.N. 99, 59I, 1939) separates these lines into three groups: (I) the yellow line at 5694; (2) the lines of 3388, 51x6, 5303, 6702, 7059, 8024, I0,749 and 10,798 ; and (3) the 6374 and 7892 lines. When his photometric measures are more precise, Lyot believes that it will be possible for him to find differences between the rays of a single group and to classify them in order according to decreasing ionization potentials.

During the 3-day interval at the time of the total eclipse of July 9, r945, Waldmeier (loc. cit.) was able to come to valuable conclusions derived from his excellent observations. The variations in intensity of the 5303 and $6374 \mathrm{~A}$. lines along the Sun's limb show that the maxima displayed may be classified into three groups: (I) those belonging to the spot zone of the old cycle, (2) those belonging to the new cycle, and (3) those belonging to the zone of activity in high latitudes. The greatest intensity of the 5303 line is equal to $5 \times 10^{-5} \mathrm{~A}$. equivalent breadth of the spectrum at the Sun's equator and it decreases to one-sixth of this value at $2^{\prime} \cdot 74$ from the Sun's limb. The intensity of the continuous spectrum of the corona at the point of its greatest intensity at an angular distance $0^{\prime} \cdot 4$ from the Sun's limb is equal to $7 \cdot 8$ millionths of the intensity at the Sun's centre. The position of greatest intensity in the coronal spectrum takes place at about the same 
elevation above the Sun where the strongest hydrogen lines, or $\mathrm{H}$ and $\mathrm{K}$ of $\mathrm{Ca}$ II, fade off to insignificance.

The Spectrum of the Corona. There are three distinct spectra:

(a) The emission spectrum existing close to the Sun's edge consisting of 23 well-determined lines between the wave-lengths 3328 and ro,798 A.

(b) A continuous spectrum of intensity JK, in the inner corona.

(c) A faint Fraunhofer spectrum of intensity JF in the outer corona. The emission spectrum is superposed on a strong continuous background and it is always very difficult for an observer to decide whether or not he can be sure of a feeble line on his photographs. In Lick Obs. Bull. ro, I, Igr8, Campbell and Moore listed 36 coronal lines between 3328 and $6374 \mathrm{~A}$. Nineteen of these lines have not been confirmed. At the I936 eclipse, the Japanese astronomers (Trans. I.A.U. 6, 75, I938) measured several feeble lines and, at the same eclipse, Petrie and Menzel ( $A p . J .96,395$, I942) measured ten additional lines. The reality of these faint lines must be confirmed (M.N. 105, 323, I945).

Emission Spectrum of the Corona. In I94I, Edlen astounded the astronomical world by giving identifications for 20 of the 23 known coronal lines; all of the transitions represent 'forbidden' lines. The green line, 5303, is ascribed to Fe XIV and the red line, 6374, to $\mathrm{Fe} \mathrm{X}$. Two of the fainter lines, 4359 and $5536 \mathrm{~A}$., are assigned to argon, an element that does not appear in the chromosphere. The stripping of atoms of Io to I5 electrons, or of half their normal electron envelope, demands a temperature of about one million degrees. Several authors have enumerated the arguments for assuming this colossal temperature. These arguments are:

(I) The high ionization of $\mathrm{Fe}, \mathrm{Ni}, \mathrm{Ca}$ and $\mathrm{A}$.

(2) The width of the emission lines if due to thermal Doppler effect.

(3) The blurring of the Fraunhofer lines in the continuous spectrum of the inner corona assumed to be the effect of the velocities of the scattering atoms.

(4) The absence of the Balmer lines in the emission spectrum explained by the electrons moving too fast to be captured.

(5) Great thermal velocities are required to explain the electron concentration as a function of the distance to the Sun.

In the chromosphere conflicting information regarding temperatures comes from excitation, line profiles and density gradients, while, on the contrary, in the corona all the methods assign the same high temperatures. There have been many plausible guesses at the source of these high temperatures, and the most encouraging explanation so far is that of Woolley and Gascoigne (M.N. ro6, II3, I946).

Regarding the problems of temperature, Bowen and Swings ( $A p . J$. ro5, 92, 1947) state that from the astrophysical standpoint, the presence in the coronal spectrum of lines in the roth to I 5 th stages of ionization has raised many difficult questions. Particularly disturbing is the presence of very highly ionized atoms in the corona, while in the photosphere, chromosphere and prominences there are neutral and singly ionized atoms only and with all intermediate stages of ionization seemingly absent from all spectra.

Perhaps the greatest solar problem to-day is to account for the forces which: (I) support prominences in general and produce the amazing prominence motions revealed by the coronagraphs and the spectroheliographs; (2) eject particles from the Sun at velocities around Iooo $\mathrm{km}$. $/ \mathrm{sec}$. to produce geomagnetic storms; and (3) produce the extended coronal streamers which very strongly give the impression of outward stream motions. Ironically enough, one difficulty will be to explain why the corona is not more extended because the million degree temperature is enough to account for the extension without further force.

Inner and Outer Corona. Grotrian (Z.f. Ast. 8, I24, I933) gave our present accepted explanation of the corona, namely, that the inner corona exhibits a continuous spectrum without dark lines and with an intensity $\mathrm{JK}$, while the outer corona, of intensity JF, shows the Fraunhofer spectrum. Moore (P.A.S.P. 35, 60, I923) showed that, even though the Fraunhofer lines were weak, they had the same widths as corresponding lines in the solar spectrum. The colour of the corona is exactly the same as that of the Sun and at all 
wave-lengths. The continuous spectrum $\mathrm{K}$ is caused by electron scattering and the spectrum $F$ is caused by the scattering from small particles in the neighbourhood of the Sun. According to Öhman (Pop. Astr. Tidskr. 27, 133, I946), the chief characteristics of the two components of the corona are:

$\mathrm{K}$ component

No spectral lines
Steep decrease in intensity outward
Same colour as the Sun
Much stronger near the Sun's equator
than near the Sun's poles

F component

Fraunhofer lines
Slow decrease in intensity outward
Same colour as the Sun
Equally bright in polar and equa-
torial regions

Two methods are possible to separate the $\mathrm{K}$ and $\mathrm{F}$ components: ( $\mathrm{I}$ ) measurement of line profiles of the Fraunhofer lines in the coronal spectrum, and (2) polarization measurements. From spectra obtained at the I940 eclipse, Allen (M.N. ro6, I37, I946) follows the spectroscopic method, while Ohman (Stockholm Obs. Ann. r5, No. 2, I947) discusses the polarization of the corona from observations made at the I 945 eclipse. Another important publication by van de Hulst $(A p . J$. 105, 47I, I947), which appeared at about the same time as the other two papers, discusses 'zodiacal light in the corona'.

As an illustration of the difficulty of measuring faint spectral lines, Allen gives measures by three observers of the same spectra. The Fraunhofer lines of the $\mathrm{F}$ spectrum gave displacements of $+0.13 \mathrm{~A} .,+0.06 \mathrm{~A}$. and $-0.06 \mathrm{~A}$., which weighted and combined gave a displacement of $+0.084 \pm 0.04 \mathrm{~A}$., equivalent to a radial velocity of $6 \mathrm{~km}$. $/ \mathrm{sec}$. This is a much smaller value than that of $20 \mathrm{~km}$./sec. derived by Moore (P.A.S.P. 35, 333, 1923; 45, I47, I933). It is evident that motions in the outer corona away from the Sun need further confirmation, especially in view of Lyot's observations which show no outward motions.

The Polarization of the Corona. By the use of instruments of four different types, Ohman was able to come to important conclusions concerning the $K$ component of the corona: (I) There is no change in the colour of the corona when proceeding from the Sun's limb outwards. (2) There is no run in polarization with wave-length. (3) The polarization at the poles is considerably smaller than the polarization at the equator. (4) At the equator, the degree of polarization has a maximum value of $45.5 \%$ at 0.6 radii from the edge of the Sun and decreases at greater distances from the limb.

Future Coronal Problems. In order to further substantiate Edlén's interpretation of the emission spectrum, it is desirable to secure wave-lengths of additional faint coronal lines. This may be accomplished only if an efficient spectrograph of great light-gathering power is employed.

It is very gratifying to realize that the three most recent publications by Allen, by Ohman and by van de Hulst reach conclusions in substantial agreement. The brightest parts of the inner corona result from the $\mathrm{K}$ corona caused by electron scattering. The rays and streamers are always prominent features in all eclipse photographs, and they must consist of concentrations of electrons. The shape of these streamers determines the ellipticity of the corona, whose shape is closely connected with the sunspot cycle (Hand. $\operatorname{der} A$ stroph. 7, 396, I936). The observations by Allen of isophotes of the I940 eclipse give the ellipticity of the corona in the usual Ludendorff notation as $a+b=0 \cdot 07+0 \cdot 13$.

Particularly important at future eclipses will be photographs taken in the light of the $\mathrm{K}$ line, through the use of a monochromatic filter which presumably would tend to remove the Fraunhofer component of the corona or at least reduce it. By this means it would be possible to photograph streamers at greater distances from the Sun. How are these streamers with the plumes and arches shown on eclipse photographs related to the coronagraph photographs in the emission light of 5303 and 6374 A.? The structures in the green and red lines resemble each other in so few details, and in turn they have so few connections with prominences that it will be most important at future eclipses for intensive work to be accomplished by coronagraphs at their own installations, as was done by Waldmeier at the r945 eclipse. 
Although the coronagraphs working outside of an eclipse can give valuable information regarding the brightest parts of the corona, the $\mathrm{K}$ corona, the faint outer $\mathrm{F}$ corona will remain exclusively an eclipse phenomenon. This corona must be circular in outline and in all probability its intensity is fairly constant though there may be changes in brightness during the sunspot cycle on account of variations in the accumulations of solid particles in the neighbourhood of the Sun.

Further eclipse observations are desirable in order to measure the profiles of the Fraunhofer lines to ascertain whether these lines have widths identical with the corresponding lines in the solar spectrum. Although a most difficult undertaking, it is urgently necessary to redetermine any motions outward in the outer corona. Photographs secured by the Russians at the I936 eclipse $(M . N$. I04, I40, I944) from four widely separated stations, show no outward motions. Allen's I940 eclipse measures are interpreted by him to indicate no motions. Lyot interprets his own coronagraph observation as confirming no motions.

The publications by Allen and by van de Hulst are in complete agreement in ascribing the Fraunhofer component of the corona to diffraction by solid particles similar to those forming the zodiacal light. It is therefore necessary to emphasize again what was published in the 1938 report of Commission I3, namely, the desirability of observing the zodiacal light at the time of a total eclipse, both by photographs with cameras probably of the Schmidt type and by visual observations. It seems possible that the visual observations by a trained observer will give the more satisfactory results.

Solar Radio Noise. Recent eclipses have added a new field of investigation dealing with the ionosphere, which may be carried out with equal success whether the skies at eclipse time are clear or cloudy. Up to the present these observations have largely been made by radio technicians, but probably in the future more astronomers will become active in the observational programme. The techniques devised in the Second World War and the extensive use of airplanes for eclipse observations should lead to a rapid accumulation of observations, while the million-degree temperature of the corona should provide a sufficiently high temperature for the theorists.

Ionospheric Problems. What are these active areas that may explain the ultra-highfrequency waves from the Sun, in the range of 30-I000 Mcyc.? According to the observations made up to the present time, it is believed that radio noise results from violent disturbances on the Sun that are associated with sunspots. It must be remembered, however, that sunspots are cooler than the photosphere, while in contrast the milliondegree corona furnishes the theorists with an important tool of research. Several publications have appeared, including one by Kiepenheuer (Ann. d. Astrophys. 9, 42, 57, I946). Results from the I940 eclipse (M.N. 103, 24, I942) showed evidence of the ultra-violet eclipse in all three regions, E, F I and $\mathrm{F}_{2}$, but there was no evidence of a particle eclipse. Interpretation led to the conclusion that the radiation producing the ionosphere was not distributed equally over the Sun's surface but was localized in the active areas. In recent years, several partial eclipses have been observed with ionospheric equipment. These partial eclipses may be used for determining the recombination coefficient in the various ionospheric layers and the distribution over the Sun of the ultra-violet source which produces the ionosphere.

Outstanding Solar Problems. The most perplexing problems are those involved with temperatures. In the chromosphere, the excitation temperature is about $5000^{\circ}$, the kinetic temperature determined from line profiles and the ionization temperature determined from HeII are about $30,000^{\circ}$, while the temperatures derived from density gradients are still higher. At what distance above the Sun do these discordant temperatures change to the million-degree temperature of the corona? What is the mechanism that can produce the amazing motions exhibited by the prominences? What is the angular relativity displacement at the Sun's edge?

The Relativity Displacement. Practically no additional observational material has come from recent eclipse expeditions. Results from previous expeditions are not in satisfactory accord, with the observed values generally higher than that predicted. At the May 20, 
I947 eclipse, Van Biesbroeck was successful with his clever device of placing in front of the lens of his 20 -foot telescope and at a $45^{\circ}$ angle, a plane-parallel optical plate halfcoated in order to bring in an auxiliary star-field $90^{\circ}$ from the Sun and at the same zenith distance. At eclipse time the sky was quite favourable, and in August additional plates were taken of the check-field. Unfortunately, the eclipse star-field in I947 was in a dark lane in the constellation Taurus. Van Biesbroeck writes: 'On the eclipse plates, only 5 stars could be measured within a distance of 4 solar radii. The majority of the 46 others were so far removed that they hardly contribute to the weight of the determination. The faintest star used is Io*0 photovisual magnitude.'

Geodetic Observations at Eclipses. The geodetic problem of Banachiewiez and Bonsdorff (Observatory, 66, 77, I945) is essentially a Scandinavian project. Therefore, it was fortunate that a total eclipse occurred so near home in July 9, I945. The success of the research depends on very accurate determination of the times of second and third contacts. The highest accuracy may be attained by photographic observations of the flash spectrum. Quoting from a letter from Lindblad: "The photometry of the continuous photospheric spectrum gives a photometric determination of the lunar contour and a very accurate connection between this contour and the photosphere at a well-defined instant. The rate of decrease of the intensity at second contact is about 5 mag. per second of arc in the blue and violet and is in fair agreement with theoretical predictions by Wildt and Waldmeier.' The undeniable success of this geodetie scheme in I945 made it desirable to continue the observations in 1947. Accordingly, Finland sent two expeditions, one to Bocayuva in Brazil, and one to Kpong on the Gold Coast. The Swedish expeditions were located in French Togo and at Araxá in Brazil. Three of the four expeditions had clear skies; the failure was in Araxá, where Ohman had planned polarization observations in addition to the geodetic plans. The Swedish expedition was one out of seven at Araxá that experienced bad luck from clouds. The results from the geodetic observations will be awaited with interest.

Coming Total Eclipses. The total eclipses of the future will in general require long distances of travel away from home.

\begin{tabular}{lcl} 
Date & $\begin{array}{c}\text { Duration } \\
\text { in minutes }\end{array}$ & \multicolumn{1}{c}{ Where visible } \\
1948, Nov. 1 & $1 \cdot 9$ & Central Africa, Madagascar \\
1950, Sept. 12 & $0 \cdot 9$ & North-eastern Siberia \\
1952, Feb. 25 & $3 \cdot 0$ & Equatorial Africa, Red Sea, Arabia, Siberia \\
1954, June 30 & $2 \cdot 5$ & Canada, Scandinavia, Russia \\
1955, June 20 & $7 \cdot 2$ & Ceylon, Indo-China, Philippines
\end{tabular}

It would seem desirable to transfer the work of the coronagraphs from Commission I3 to Commission II or to Commission I2.

\author{
S. A. Mitchell \\ President of the Commission
}

\title{
Report of meeting
}

President: Prof. S. A. Mitchell.

Views expressed on new problems in coronal spectroscopy were as follows. There is need for extensive laboratory measurements before the identification of coronal lines can be advanced much further. The spectral distribution in the continuous coronal spectrum requires confirmation at forthcoming eclipses, and further search for faint emission lines is recommended.

The suggestion that the outer corona is caused mainly by diffraction of zodiacal light particles is in satisfactory agreement with the latest measurements of the coronal Fraunhofer lines and the polarization. It was pointed out that certain physical quantities in 
the corona, the hydrogen to metal ratio, the degree of ionization, the atomic excitation collision cross-section, and the emission of ultra-violet light, can all be determined with reasonable satisfaction. The ultra-violet emission is thought to be mainly in the principal lines of $\mathrm{MgX}$. Theoretical calculations of cross-sections have been made also, and will be available for comparison with observations.

Problems of the corona are profoundly influenced by the realization that the source of most of the solar radio-noise is located in the corona. Reports of solar noise observations were received.

Observations made with the Climax coronagraph show a most suggestive association between the emission of the line $5694 \mathrm{~A}$. and active looped prominences. The $6374 \mathrm{~A}$. line shows velocity displacements of up to $50 \mathrm{~km}$. $/ \mathrm{sec}$. in these regions.

Attention was drawn to the unsatisfactory state of our knowledge of temperature at different levels in the chromosphere. In this connection there is a call for eclipse observations of darkening at the extreme limb, and also for measurements as high as possible into the corona of both atom density gradients and the width of the lines of $\mathrm{Ca}^{+}$and $\mathrm{H}$. 